\title{
Questes
}

vestes Revue pluridisciplinaire d'études médiévales

$18 \mid 2010$

La règle du jeu

\section{La règle du jeu : avant-propos}

Jean-Michel Mehl

\section{(2) OpenEdition}

\section{Journals}

Édition électronique

URL : http://journals.openedition.org/questes/651

DOI : 10.4000/questes.651

ISSN : 2109-9472

\section{Éditeur}

Les Amis de Questes

\section{Édition imprimée}

Date de publication : 15 février 2010

Pagination : 1-3

ISSN : 2102-7188

\section{Référence électronique}

Jean-Michel Mehl, «La règle du jeu : avant-propos », Questes [En ligne], 18 | 2010, mis en ligne le 01

janvier 2014, consulté le 24 septembre 2020. URL : http://journals.openedition.org/questes/651 ; DOI : https://doi.org/10.4000/questes.651 


\title{
Avant-propos
}

\author{
Jean-Michel MEHL \\ Professeur émérite \\ Université de Strasbourg
}

On connaît le jeu de mots classique : «Porte ayant du jeu recherche partenaire ». De manière triviale, il ne fait que dire l'étonnante polysémie du terme « jeu ». Ce dernier appartient en effet à cette catégorie de mots simples qui, comme le soulignait Paul Valéry, introduisent « une résistance étrange » et déjouent « tous les effets de la définition aussitôt que vous les retirez de la circulation » pour les examiner à part. Quant aux ethnologues, ils nous apprennent qu'il y a des civilisations dans lesquelles les activités ludiques et les activités de labeur sont rendues par le même mot. Un comble alors que, dans l'acception courante, tout paraît opposer le jeu au travail! Gardons toutefois à l'esprit que, selon la remarque de Georges Gusdorf, «l'esprit du jeu peut s'introduire au cœur de l'activité qui lui semble la plus étrangère, comme un parasite qui l'habiterait secrètement, sans que de l'extérieur il en paraisse rien $»^{1}$.

Faut-il dès lors renoncer à toute définition précise du jeu? Johan Huizinga avait tendance à voir dans le jeu le point de départ de toute culture $^{2}$. Cela le conduisait à voir le jeu toujours et partout, en gommant à peu près tout ce que l'activité ludique a de spécifique. Pour sa part Jean Duvignaud sépare nettement ce qui relève, dans la terminologie anglaise, du play et du game, voyant dans le premier une activité libre et sans règle,

\footnotetext{
${ }^{1}$ Georges GuSDORF, «L'esprit des jeux », in Roger CAILlois (dir.), Jeux et sports, Encyclopédie de la Pléiade, Paris, 1967, p. 1157 -1158.

2 Johan Huizinga, Homo Ludens, essai sur la fonction sociale du jeu, traduit par Cécile SERESIA, Paris, Gallimard, 1967 (1 ${ }^{\text {ère }}$ édition en 1938).
} 
tandis que le cours du second se trouve construit par la règle ${ }^{3}$. L'insuffisance de ces deux approches est patente. C'est pourquoi la recherche de dénominateurs communs, telle que celle tentée par Roger Caillois paraît plus pertinente ${ }^{4}$. Parmi ceux-ci, la règle se détache nettement. De fait, même les jeux qui peuvent nous paraître sans règle, je songe aux jeux d'enfants, obéissent peu ou prou à des règles, sachant que la transgression peut aussi devenir règle.

Les différents textes rassemblés ici disent à l'évidence la luxuriance de la notion de jeu, tout comme l'entrelacs permanent entre le play et le game dans la société médiévale, entrelacs souligné dès les propos introductifs.

De part et d'autre de ce regroupement, le jeu d'échecs, incontestablement un game qui, pourtant, vient s'accoupler à un play dans l'épisode de la Forêt perdue dans le Lancelot en prose, paraît essentiel. En même temps, le jeu contre l'échiquier magique ne peut que faire songer au jeu actuel contre l'ordinateur, autre magie ! Conformément à ses origines, le jeu d'échecs est bien là le jeu de la mort et s'apparie à un savoir comme le souligne l'étude de ses origines troyennes. Certes, derrière cette localisation spatiale transparaît la volonté de situer au loin l'origine de pratiques considérées souvent avec suspicion par les autorités spirituelles et civiles. En même temps, replacée dans un contexte didactique, ce jeu ou en tout cas sa démarche, l'apparie à celle de l'exercice scolaire de la disputatio. Comme cette dernière, le jeu est à information complète. L'usage du mot ludus pour désigner l'école place ce thème dans la même atmosphère.

\footnotetext{
${ }^{3}$ Jean Duvignaud, Le Jeu du jeu, Paris, Balland, 1980, p. 14

${ }^{4}$ Roger CAILlois, Les Jeux et les hommes. Le masque et le vertige, Paris, Gallimard, 1958, p. 42-43.
} 
Mais ludus renvoie aussi explicitement au théâtre en tant que représentation organisée. Il est frappant de voir que les jugements à son égard accordent beaucoup d'importance aux localisations spatiales, temporelles et à la condition des joueurs, autant d'éléments qui finissent aussi par être pris en compte dans l'appréciation des autorités sur les jeux divers.

Cette règle est également au centre du genre littéraire particulier qu'est le jeu-parti. Tournoi en vers qui fait coexister plaisanteries et savoir, le jeu-parti illustre bien la nécessité du respect de la règle. Fondé sur cette dernière, le jeu offre naturellement la possibilité de la transgression de cette même règle. C'est ce qu'illustre le Lai d'Ignaure qui, mettant en pratique les bases du jeu réel du Roi qui ne ment, permet d'inverser les fondements de l'amour courtois.

Johan Huizinga disait que «tout jeu signifie quelque chose ». C'est bien ce que notifient les textes rassemblés ici. Quelque part tous renvoient à la notion de liberté, même si cette dernière est à prendre dans le sens où l'entend Colas Duflo: « Le jeu est l'invention d'une liberté dans et par une légalité $»^{5}$. À n'en pas douter tous les auteurs des textes qui suivent se sont pris au jeu!

${ }^{5}$ Colas DufLO, Jouer et philosopher, Paris, PUF, 1997, p. 57. 